\title{
An alternative to a randomized control design for assessing the efficacy and effectiveness of bracing in AIS
}

Daniel Y. T. Fong, PhD; Kenneth M. C. Cheung, MBBS, MD, FRCS, FHKCOS, FHKAM(Orth); Yat-Wa Wong, MBBS, FRCSE, FHKCOS, FHKAM(Orth); Wai-Yuen

Cheung, MBBS, FRCSE, FHKAM, FHKCOS; Idy C. Y. Fu, DN; Evelyn E. Kuong, MBBS; Kin-Cheung Mak, MBBS; Michael To, MBBS; Dino Samartzis, DSc; Keith D. K. Luk, MCh(Orth), FRCSE, FRCSG, FRACS, FHKAM(Orth)

\section{Author Affiliations}

Dr Daniel Yee Tak Fong, PhD (e-mail: dytfong@hku.hk)

- School of Nursing, The University of Hong Kong, Pokfulam, Hong Kong SAR, China

Prof Kenneth Man Chee Cheung, MBBS, MD, FRCS, FHKCOS, FHKAM(Orth)

(e-mail: cheungmc@hku.hk)

- Department of Orthopaedics and Traumatology, The University of Hong Kong, Pokfulam, Hong Kong SAR, China

Dr Yat-wa Wong, MBBS, FRCSE, FHKCOS, FHKAM(Orth) (e-mail: wongyatwa@i-cable.com)

- Department of Orthopaedics and Traumatology, The University of Hong Kong, Pokfulam, Hong Kong SAR, China

Dr Wai-Yuen Cheung, MBBS, FRCSE, FHKAM, FHKCOS (e-mail: 
lcheung2@netvigator.com)

- Department of Orthopaedics and Traumatology, The University of Hong Kong, Pokfulam, Hong Kong SAR, China

Dr Idy Chui Yuk Fu, DN (e-mail: idyfu2001@hotmail.com)

- School of Nursing, The University of Hong Kong, Pokfulam, Hong Kong SAR China

Dr Evelyn E Kuong, MBBS (e-mail: eekuong@gmail.com)

- Department of Orthopaedics and Traumatology, The University of Hong Kong, Pokfulam, Hong Kong SAR, China

Dr Kin-Cheung Mak, MBBS (e-mail:drmak.spine@gmail.com)

- Department of Orthopaedics and Traumatology, The University of Hong Kong, Pokfulam, Hong Kong SAR, China

Dr Michael To, MBBS (e-mail: mikektto@hku.hk)

- Department of Orthopaedics and Traumatology, The University of Hong Kong, Pokfulam, Hong Kong SAR, China

Dr Dino Samartzis, DSc (e-mail: dsamartzis@msn.com)

- Department of Orthopaedics and Traumatology, The University of Hong Kong, Pokfulam, Hong Kong SAR, China

Prof Keith Dip Kei Luk, MCh(Orth), FRCSE, FRCSG, FRACS, FHKAM(Orth) (e-mail: hrmoldk@hku.hk)

- Department of Orthopaedics and Traumatology, The University of Hong Kong, Pokfulam, Hong Kong SAR, China 


\section{Correspondence:}

Keith D. K. Luk

Department of Orthopaedics and Traumatology, The University of Hong Kong, 5/F

Professorial Block, Queen Mary Hospital, Hong Kong SAR, China

Telephone number: (+852) 2255-4254

Fax number: (+852) 2817-4392

E-mail address: hrmoldk@hku.hk 


\section{ABSTRACT}

Randomized controlled trials (RCTs) that assessed the efficacy of bracing for AIS suffered from small sample sizes, low compliance and willingness of participation with limited generalizability. The aim of this study was to assess the feasibility of a comprehensive cohort study for evaluating both the efficacy and effectiveness of bracing in patients with adolescent idiopathic scoliosis (AIS). AIS patients with curves at higher risk of progression were invited to join a RCT. Those who declined were given the option to remain in the study, and to choose whether they wished to be braced or observed. A randomization schedule was generated for all patients whether or not they joined the RCT; thus, some patients who made their own choice fit with the randomized assignments. Patients were followed up every 4 months. Of 87 eligible patients identified over one year, 68 (78\%) patients with mean age of 12.5 years (range, 10-15 years) consented to participate, with a median follow-up of 77 weeks. Of these, 19 (28\%) patients accepted randomization. Of those who declined randomization, 18 (37\%) chose brace. Patients who were more satisfied with their image were more likely to have bracing chosen $(\mathrm{OR}=4.1 ; 95 \% \mathrm{CI}, 1.1-15.0 ; \mathrm{P}=0.035)$. Braced patients had an average of 17 hours in brace each day for the first year. This comprehensive cohort study design facilitates the assessment of both efficacy and effectiveness of bracing in AIS patients, which is not feasible in a conventional RCT. 


\section{Introduction}

Bracing has a long history in the treatment of adolescent idiopathic scoliosis (AIS)

patients who present radiographically with coronal curves at high risk of progression.

However, reports are mixed on whether bracing reduces curve progression and reduces

the need for surgery. ${ }^{1-5}$ A well-known prospective cohort study performed by the

Scoliosis Research Society (SRS) reported that bracing could prevent curve progression

better than observation alone ${ }^{6}$. The study was well conducted but was not randomized

with concurrent controls. In addition, there have been 5 systematic reviews or

meta-analyses of bracing, which included at most one grossly underpowered randomized

controlled trial (RCT). ${ }^{1-5,7}$ Since RCT study designs typically represent level 1 evidence

to assess the therapeutic utility of an intervention, to our knowledge, only two large

multi-center RCTs have assessed the efficacy of bracing for AIS and are no longer

recruiting patients. ${ }^{8,9}$ However, both trials encountered recruitment difficulties. ${ }^{10}$

Although a RCT design is regarded as the "ideal" for establishing efficacy, it has

limitations when applied to assess the "effectiveness" of bracing in practice. This is the

case because patients, their parents, and physicians cannot be blinded to bracing. Thus,

preferences and expectations may influence the effectiveness of bracing through various

poorly understood pathways. ${ }^{11,12}$ Secondly, cultural, social, and economic factors might

affect decisions by patients/parents to participate in a RCT. Indeed, there have been 
reports of very poor participation in RCT, which severely limit the generalizability of results obtained. ${ }^{10,13}$ It is therefore important to determine what patients and their families think of the available therapeutic options for AIS, which again cannot be addressed in a traditional RCT design. ${ }^{14}$

In view of the paucity of RCTs assessing the efficacy of bracing for AIS and the low acceptance of AIS patients to consent to enter a RCT, we proposed an alternative design to evaluate both the efficacy and effectiveness of bracing over observation. To properly plan this newly designed study, we conducted a feasibility study to gain preliminary scientific and logistic information.

\section{Patients and Methods}

\section{Design}

This was a comprehensive cohort study (CCS) in which treatment with bracing or observation was determined either by randomization or by the preference of the patients/parents (Fig. 1). Eligible patients were followed up every 4 months for a minimum of 12 months. Ethics approval was granted by a local institutional review board (Study Identifier in ClinicalTrials.gov registry: NCT00989495).

The CCS design is a new paradigm of evidence-based clinical research that combines a RCT and a preference-based controlled trial. ${ }^{11}$ The treatment allocation plan 
is shown in Fig. 1. The trial examines the treatment efficacy as in a traditional RCT

(Groups I versus II in Fig. 1); it also addresses the issue of effectiveness by assessing the impact of treatment preference (Groups I versus II and Groups III+IV versus IV+VI in Fig. 1). Because of the concurrent assessment of efficacy and effectiveness, the inclusion of non-randomized subjects has even been suggested in all RCTs to assess external validity. ${ }^{15}$ Moreover, the CCS design has been previously adopted in major cancer trials. $^{16,17}$

\section{Study Subjects}

Patients aged 10 years or above, with Risser sign $0-2$, and diagnosed with AIS with either (1) a Cobb’s angle of $20^{\circ}$ to $<25^{\circ}$ and at least $5^{\circ}$ deterioration over the past 4 months, or (2) a Cobb’s angle of $25^{\circ}$ to $<30^{\circ}$, were invited to participate. Excluded patients were those with a history of treatment for AIS, who were $\geq 1$ year postmenarchal (girls only), who had physical or mental disability that interfered with adherence to bracing, with diagnosed musculoskeletal or developmental illness that might be responsible for the spinal curvature, with structural thoracic scoliosis with apex above T7, who had difficulty understanding or completing study questionnaires, or who were unlikely to comply with the study protocol. These criteria mostly conformed with the criteria suggested by the SRS for bracing studies. ${ }^{18}$ We planned to recruit at least 10 
patients in each of the two RCT groups and at least 5 patients in each of the other four groups (Fig. 1).

\section{Interventions}

For patients who underwent bracing, a custom-made thoraco-lumbar sacral orthosis (TLSO) was molded under traction by an experienced orthotist. The brace extended anteriorly from the pubis to the xiphoid process. Patients were asked to wear the brace for a minimum of 20 hours per day, and the brace straps were marked to ensure that correct pressure was applied. Patients under observation only returned for assessment as scheduled.

\section{Study Procedures}

Before patient recruitment, a treatment allocation schedule was generated by block randomization with a block size randomized as 4 or 8 . The allocation sequence was concealed using opaque envelopes, each containing the allocation code of 1 patient. Patients were recruited at the outpatient clinic of a specialist hospital, a children's hospital largely devoted to orthopedic patients and run by our investigators under standardized protocols. There were 2 clinic sessions per week. Before each clinic session, a recruitment team composed of a research assistant and 2 authors [DF] and [IF] 
screened all patient records and tagged potential cases. During a clinic session, tagged patients were seen by orthopedic surgeons to assess study eligibility. All these surgeons were given the study protocol and explained of the study procedures before they performed the eligibility check. Patients with confirmed eligibility were referred to the recruitment team, who explained the study details and sought informed consent. The research assistant independently performed patient recruitment and follow-up after six months of coaching by [DF] or [IF]. All study patients had medical services provided by surgeons belonging to our study team.

Each eligible patient was first invited to participate in a RCT. Those who declined were given an option to stay in the study and choose whether they wished to be braced or observed. All patients and their parents were given time and allowed to consult anyone, including their orthopedists, before they decided. If an eligible patient sought advice from an orthopedist in the specialist hospital, the orthopedist would remain impartial regarding brace or observation, and would only advise that because of insufficient evidence to demonstrate the effectiveness of bracing. Patients would decide their preferred treatment at the end. Consenting patients signed a consent form together with one of their accompanying guardians or parents. The 60 patients with the best study compliance received about US\$25.

Braced patients were called at least 1 day before their next clinical visit to remind 
them to remove the brace at least $24 \mathrm{~h}$ before the visit. All study deviations were

recorded for all study patients.

\section{Measurements}

In all study patients, socio-demographics were recorded at baseline. At all clinical visits, height $(\mathrm{cm})$, weight $(\mathrm{kg})$, arm span $(\mathrm{cm})$, menarche status (girls only), and exercise habits were measured. Moreover, participants self-completed the Scoliosis Research Society-22 (SRS-22) questionnaire, the State-Trait Anxiety Inventory (STAI), and the Beck Depression Inventory version II (BDI-II). These tools have been demonstrated with good validity and reliability in Chinese subjects. ${ }^{19-21}$ In addition, standing posteroanterior out-of-brace radiographs of the full spine were taken. An orthopaedic surgeon [EK] performed blinded assessment of the radiographs without knowing patient treatment status. Braced patients were also given a diary to record the daily number of hours in brace and asked to return completed diaries at each follow-up visit. An accompanying parent of each patient also self-completed the STAI and BDI-II.

\section{Statistical Analysis}

Quality of life measures were scored in accordance with the corresponding scoring

manuals. ${ }^{22,23}$ Specifically, the SRS-22 has domains scored as means in the range of 1 to 
5, according to the Scoliosis Research Society. If fewer than half of the items in a domain had responses, the skipped items were scored by the mean of the other items in the domain. Otherwise, the domain score was recorded as missing.

Determinants of acceptance to randomization were examined by comparing groups I+II and III+IV+V+VI (described in Fig. 1) by a stepwise logistic regression on all baseline measurements. Model goodness-of-fit was assessed by the Hosmer-Lemeshow test. All significance tests were two-tailed, with 5\% used as the level of significance. Analysis was performed using Statistical Analysis System (SAS) Version 9.2 (Cary, NC).

\section{Results}

Between March 9, 2009 and March 8, 2010, 87 eligible AIS patients were approached and $68(78 \%, 95 \%$ CI = 68 to 86\%) consented to participate in the study. On average, 5.7 patients were recruited per month.

Among the 19 refusals, 16 (84\%) were made by the parents (11 mothers and 5 fathers) and 3 were made by the children (Fig. 1). Parents refused to participate because they were unwilling to participate in a research study $(n=7)$, wanted to decide at the next visit $(n=6)$, were in a hurry $(n=2)$, or thought that we could do a retrospective study without consent $(n=1)$. One newly diagnosed girl was too emotionally unstable to 
participate. Another girl whose mother consented to randomization was afraid of bracing and refused to participate. The other boy refused participation because he was unwilling to complete the questionnaires.

Of the 68 study patients ( 5 boys and 63 girls), 19 (28\%, 95\% CI = 17.8 to $40.1 \%)$ consented to be randomized (Table I). The participation rate in the RCT was 21\% (95\% $\mathrm{CI}=12.7$ to $30.7 \%)$. Among the 49 patients who chose their own treatment, 18 (37\%, 95\% CI $=23.4$ to 51.7\%) chose bracing (Table II). Taller patients $(\mathrm{OR}=0.90,95 \% \mathrm{CI}=$ 0.81 to $0.995, \mathrm{p}=0.040)$ or those who had higher monthly household income $(\mathrm{OR}=$ $0.75,95 \% \mathrm{CI}=0.57$ to $0.98, \mathrm{p}=0.032$ ) preferred to choose their treatment preference (Table III). Among patients who had their treatment preferences, those with one unit score higher in the satisfaction with self-image domain were 3 times more likely to choose bracing $(\mathrm{OR}=4.1,95 \% \mathrm{CI}=1.1$ to $15.0, \mathrm{p}=0.035)$ (Table III).

Patients were followed for a median of 77 weeks (range $=0$ to 128 weeks), and 6 withdrew from the study within the first year of follow-up. The parents of 2 refused study continuation because of unwillingness to complete study questionnaires. Another 3 did not continue follow-up at the specialist hospital after recruitment. The other 2 patients did not provide reasons for withdrawal.

Of 29 braced patients, 27 (93\%) returned their diaries. The overall mean number of hours in brace during the first year was 17.0 per day (95\% CI = 15.5 to 18.6), which was 
not significantly different between those randomized and those who chose to be braced $(p=0.634)$.

Ten patients had $\geq 6$ degrees of curve progression during follow-up. All of them chose their own treatment with two of them chose bracing. The risk of curve progression $\geq 6$ degrees in patients who chose bracing was $51 \%$ of that in patients who chose observation $(\mathrm{HR}=0.51,95 \% \mathrm{CI}=0.10$ to $2.69, \mathrm{p}=0.426)$ despite it was insignificant due to small sample size.

\section{Discussion}

The CCS design addresses the concern of limited generalizability in traditional RCTs for bracing due to the low acceptance to treatment decided by randomization. The participation rate in our RCT component was 21\%, the same as that in a Dutch RCT but lower than the $33 \%$ reported in a hypothetical setting ${ }^{10,13}$. Refusing to consent to enter a RCT as a result of strong patients' preference on their treatment is observed in this study. The potential influence of such preference on brace effects can be addressed by including the preference component under our CCS. This is what a traditional RCT cannot accomplish.

The analysis of a CCS should begin with an assessment of efficacy of bracing by comparing the brace and observation groups under the RCT component, i.e. Groups I 
versus II. Then, characteristics such as demographics and socio-economic status of patients between the RCT and preference components are compared to identify determinants of treatment preference, i.e. Groups I+II versus III+IV+V+VI. Finally, the impact of treatment preference on brace effects is examined by assessing the preference by treatment interaction, i.e. (Groups I versus II) and (Groups III+V versus IV+VI) ${ }^{24}$. When there is no indication of an influence of treatment preference, a more reliable estimate of brace effects based on patients in the two components can be obtained.

Taller patients or those with higher monthly household income were more likely to choose their desired treatment rather than relying on chance. The treatment decision was mostly made by the patients’ parents, and most chose observation. Parents of taller patients think their children have little potential to get taller, and thus they were more determined to choose observation. On the other hand, parents with higher financial resources were often more educated and had a better understanding of the risks and benefits of bracing. They were more prone to express preferences and to refuse allocation of treatment by randomization.

It is interesting to note that higher satisfaction of the patients with their image could influence their parents to choose bracing. A main consideration of parents was whether their children would accept bracing, which has previously been shown to lower the satisfaction of patients with their image ${ }^{25}$. It could be that patients who had higher 
satisfaction with their image have a higher tolerance of their appearance and thus are more open to bracing. On the other hand, the satisfaction level with image in patients who chose observation, although lower than that in patients who chose bracing, was still good with a fairly high mean score of 3.7 in the range of 1 to 5 . These patients did not exhibit significant dissatisfaction with their curvature, to the extent that it would make them choose to be braced to improve their appearance.

Several important aspects must be considered in planning for the full-scale CCS. First, during patient recruitment, it is important to have all orthopedists and patient recruiters to be clear regarding the lack of good evidence in support of bracing for AIS patients at their degree of curvature. We recruited AIS patients at a higher risk of curve progression but with curvature less than 30 degrees. In this selected group of patients, most orthopedists would be comfortable with observation only. Nevertheless, most if not all parents would like to have the best treatment for their children. During the consent process, many parents wanted the orthopedists and recruiters to make the best treatment decision for them. Subjective opinions of orthopedists or recruiters towards a treatment would lower acceptance of randomization and thus limit the generalizability of the RCT. Second, there were unbalanced numbers of subjects across the different treatment groups. Although the unbalanced group sizes would not alter the estimated treatment effects, balanced group sizes would result in smaller standard errors, and hence larger 
power to detect an effect, when the sample size remains unchanged. Hence, block randomization stratified by whether patients consent to randomization is recommended. This also enables the two randomized groups to be considered as a RCT on their own. Third, it is preferable to allow full relaxation of the spine to facilitate a more realistic measure of the curvature. Our braced patients were called at least 24 hours before their next clinical visit to remind them to remove braces at least 24 hours before the visit. Additionally, clinic orthopedists were informed that out-of-brace x-rays were needed in study patients.

When compared with a traditional RCT, a CCS additionally recruits patients who do not consent to randomization for assessing the bracing effectiveness. Since around $70 \%$ of our study patients received their desired treatment, the sample size in a CCS may triple that of a traditional RCT. However, study completion time should be similar since a CCS would also need to ensure sufficient patients for its RCT component.

In a similar study design by Weinstein et al which started recruitment of subjects after our initial study recruitment, the authors noted the efficacy of bracing in AIS subjects and terminated their study prematurely due to the effect size of bracing over observation in decreasing curve progression ${ }^{26}$. Similar to our study, the authors further noted the difficulty in recruiting patients for their RCT arm of the study and the inclination for patients to choose treatment-type. However, their study population was 
based on a heterogeneous western culture and not on an Asian population, which may also present with specific ethnic-considerations related to study and treatment selection as well as outcomes.

Our study is the first to definitely demonstrate that there is an inability in blinding patients, parents, and physicians to brace treatment and the strong preference of parents in choosing their desired treatment may limit the generalizability of traditional RCTs. Furthermore, we have successfully completed a feasibility study of a CCS design that additionally allows the assessment of the impact of preference on the effectiveness of bracing. The study design is feasible, and a full-scale trial is being planned, whose model can be further utilized to address the efficacy and effectiveness between different ethnic/cultural populations. 


\section{References}

1. Lenssinck ML, Frijlink AC, Berger MY, Bierman-Zeinstra SM, Verkerk K, Verhagen AP. Effect of bracing and other conservative interventions in the treatment of idiopathic scoliosis in adolescents: a systematic review of clinical trials. Phys Ther. Dec 2005;85(12):1329-1339.

2. Dolan LA, Weinstein SL. Surgical rates after observation and bracing for adolescent idiopathic scoliosis: an evidence-based review. Spine. Sep 1 2007;32(19 Suppl):S91-S100.

3. Maruyama T. Bracing adolescent idiopathic scoliosis: a systematic review of the literature of effective conservative treatment looking for end results 5 years after weaning. Disabil Rehabil. 2008;30(10):786-791.

4. Maruyama T, Grivas TB, Kaspiris A. Effectiveness and outcomes of brace treatment: a systematic review. Physiother Theory Pract. Jan 2011;27(1):26-42.

5. Rowe DE, Bernstein SM, Riddick MF, Adler F, Emans JB, Gardner-Bonneau D. A meta-analysis of the efficacy of non-operative treatments for idiopathic scoliosis. The Journal of Bone and Joint Surgery. May 1997;79(5):664-674.

6. Nachemson AL, Peterson LE. Effectiveness of treatment with a brace in girls who have adolescent idiopathic scoliosis. A prospective, controlled study based on data from the Brace Study of the Scoliosis Research Society. The Journal of 
Bone and Joint Surgery. Jun 1995;77(6):815-822.

7. el-Sayyad M, Conine TA. Effect of exercise, bracing and electrical surface stimulation on idiopathic scoliosis: a preliminary study. International Journal of Rehabilitation Research. Mar 1994;17(1):70-74.

8. Bunge EM, de Koning HJ. Bracing patients with idiopathic scoliosis: design of the Dutch randomized controlled treatment trial. BMC Musculoskelet Disord. 2008;9:1-7.

9. Weinstein SL, Dolan LA. Bracing in adolescent idiopathic scoliosis trial (BrAIST). 2007; http://clinicalTrials.gov.

10. Bunge EM, Habbema JD, de Koning HJ. A randomised controlled trial on the effectiveness of bracing patients with idiopathic scoliosis: failure to include patients and lessons to be learnt. European Spine Journal. May 2010;19(5):747-753.

11. Millat B, Borie F, Fingerhut A. Patient's preference and randomization: new paradigm of evidence-based clinical research. World J Surg. May 2005;29(5):596-600.

12. Grufferman S. Complexity and the Hawthorne effect in community trials. Epidemiology. May 1999;10(3):209-210.

13. Dolan LA, Sabesan V, Weinstein SL, Spratt KF. Preference assessment of 
recruitment into a randomized trial for adolescent idiopathic scoliosis. The Journal of Bone and Joint Surgery. Dec 2008;90(12):2594-2605.

14. Negrini S, Carabalona R. Social acceptability of treatments for adolescent idiopathic scoliosis: a cross-sectional study. Scoliosis. 2006;1:14.

15. Fielding LP, Grace R, Hittinger R. Patients who are eligible but not randomised should be included as additional comparative arm in study (Letters). Brit Med J. 1999;318:894-895.

16. Moergel M, Jahn-Eimermacher A, Krummenauer F, et al. Effectiveness of adjuvant radiotherapy in patients with oropharyngeal and floor of mouth squamous cell carcinoma and concomitant histological verification of singular ipsilateral cervical lymph node metastasis (pN1-state)--a prospective multicenter randomized controlled clinical trial using a comprehensive cohort design. Trials. 2009;10:118.

17. Prescott RJ, Kunkler IH, Williams LJ, et al. A randomised controlled trial of postoperative radiotherapy following breast-conserving surgery in a minimum-risk older population. The PRIME trial. Health Technol Asses. Aug 2007;11(31):1-184.

18. Richards BS, Bernstein RM, D'Amato CR, Thompson GH. Standardization of criteria for adolescent idiopathic scoliosis brace studies: SRS Committee on 
Bracing and Nonoperative Management. Spine. Sep 15 2005;30(18):2068-2075.

19. Cheung KM, Senkoylu A, Alanay A, Genc Y, Lau S, Luk KD. Reliability and concurrent validity of the adapted Chinese version of Scoliosis Research Society-22 (SRS-22) questionnaire. Spine. May 1 2007;32(10):1141-1145.

20. Shek DT. The Chinese version of the State-Trait Anxiety Inventory: its relationship to different measures of psychological well-being. J Clin Psychol. May 1993;49(3):349-358.

21. Byrne BM, Stewart SM, Lee PWH. Validating the Beck Depression Inventory-II for Hong Kong Community Adolescents. International Journal of Testing. 2004;4:199-216.

22. Spielberger CD. State-Trait Anxiety Inventory for adults: Sampler set: Manual, test booklet and scoring key. Redwood City, CA: Mind Garden, Inc.; 1983.

23. Beck AT, Steer RA, G.K. B. BDI-II Manual. New York: The Psychological Corporation; 1996.

24. Olschewski M, Schumacher M, Davis KB. Analysis of randomized and nonrandomized patients in clinical trials using the comprehensive cohort follow-up study design. Control Clin Trials. Jun 1992;13(3):226-239.

25. Cheung KM, Cheng EY, Chan SC, Yeung KW, Luk KD. Outcome assessment of bracing in adolescent idiopathic scoliosis by the use of the SRS-22 questionnaire. 
Int Orthop. Aug 2007;31(4):507-511.

26. Weinstein SL, Dolan LA, Wright JG, Dobbs MB. Effects of bracing in adolescents with idiopathic scoliosis. The New England journal of medicine. Oct 17 2013;369(16):1512-1521.

\section{Acknowledgment}

The work was financially supported by the Scoliosis Research Society. We thank Sister

Leung, Ms Josephine Lam, Ms SY Lam, and all nursing staff at the Duchess of Kent

Children's Hospital for facilitating the subject recruitment. We also thank Ms NL Kwok for recruiting the patients and coordinating the study, as well as Ms Peony Wan and Mr Geoffrey Chan for following up the patients. 
Figure Legends

Fig. 1 Patient disposition. The treatment in a square bracket is the one on the randomization schedule and that otherwise is the actual. 
Table I. Characteristics of the 68 study patients and their parents

(a) Sociodemographics

\begin{tabular}{|c|c|c|c|}
\hline & $\begin{array}{c}\text { RCT } \\
\text { component } \\
(\mathrm{n}=19)\end{array}$ & $\begin{array}{c}\text { Preference } \\
\text { component } \\
(\mathrm{n}=49)\end{array}$ & p-value \\
\hline Age (years) & $12.4 \pm 1.1$ & $12.6 \pm 1.1$ & 0.383 \\
\hline Gender & & & 0.684 \\
\hline Male & $1(5.3)$ & $4(8.2)$ & \\
\hline Female & $18(94.7)$ & $45(91.8)$ & \\
\hline Educational level & & & 0.993 \\
\hline Grade 5-6 & 7 (36.8) & $18(36.7)$ & \\
\hline Grade 7-11 & $12(63.2)$ & $31(63.3)$ & \\
\hline Height (cm) & $150.9 \pm 8.0$ & $154.8 \pm 5.6$ & 0.039 \\
\hline Weight (kg) (3 missing) & $38.3 \pm 7.9$ & $41.9 \pm 8.6$ & 0.125 \\
\hline BMI (kg/m²) (3 missing) & $16.7 \pm 2.6$ & $17.4 \pm 3.1$ & 0.404 \\
\hline Arm span (cm) & $152.2 \pm 10.2$ & $155.5 \pm 7.0$ & 0.138 \\
\hline Monthly household income (HK\$) & & & 0.096 \\
\hline$<2000$ & $2(10.5)$ & $1(2.0)$ & \\
\hline 2000-5999 & $1(5.3)$ & $2(4.1)$ & \\
\hline $6000-9999$ & $2(10.5)$ & $5(10.2)$ & \\
\hline 10000-14999 & $3(15.8)$ & $3(6.1)$ & \\
\hline 15000-19999 & $4(21.1)$ & $11(22.4)$ & \\
\hline $20000-24999$ & $3(15.8)$ & $6(12.2)$ & \\
\hline 25000-29999 & $0(0)$ & $3(6.1)$ & \\
\hline 30000-39999 & $1(5.3)$ & $5(10.2)$ & \\
\hline 40000-59999 & $1(5.3)$ & $5(10.2)$ & \\
\hline$\geq 60000$ & $2(10.5)$ & $8(16.3)$ & \\
\hline Gestational age (weeks) & $39.7 \pm 1.4$ & $39.4 \pm 1.2$ & 0.393 \\
\hline Maternal age (years) & $30.3 \pm 4.3$ & $30.0 \pm 5.0$ & 0.805 \\
\hline Breastfeeding & & & 0.750 \\
\hline No & $13(68.4)$ & $34(69.4)$ & \\
\hline Yes & $6(31.6)$ & $13(26.5)$ & \\
\hline Do not know / Missing & $0(0)$ & $2(4.1 \%)$ & \\
\hline Smoking among family members & & & 0.411 \\
\hline No & $13(68.4)$ & $27(55.1)$ & \\
\hline Yes & $6(31.6)$ & $20(40.8)$ & \\
\hline Do not know / Missing & $0(0)$ & $2(4.1)$ & \\
\hline Alcohol drinking among family members & & & 0.622 \\
\hline
\end{tabular}




\begin{tabular}{|c|c|c|c|}
\hline No & $12(63.2)$ & $26(59.2)$ & \\
\hline Yes & $7(36.8)$ & $20(40.8)$ & \\
\hline Do not know / Missing & $0(0)$ & $3(6.1)$ & \\
\hline Family history of scoliosis & & & 0.223 \\
\hline No & $14(73.7)$ & $29(59.2)$ & \\
\hline Yes & $1(5.3)$ & $8(16.3)$ & \\
\hline Do not know / Missing & $4(21.1)$ & $12(24.5)$ & \\
\hline
\end{tabular}

(b) Curvature and quality of life measures

\begin{tabular}{|c|c|c|c|c|c|}
\hline & \multicolumn{2}{|c|}{$\begin{array}{c}\text { RCT } \\
\text { Component } \\
(\mathrm{n}=19)\end{array}$} & \multicolumn{2}{|c|}{$\begin{array}{c}\text { Preference } \\
\text { component } \\
(\mathrm{n}=49)\end{array}$} & \multirow[b]{2}{*}{ p-value } \\
\hline & $\mathrm{n}$ & mean \pm SD & $\mathrm{n}$ & mean \pm SD & \\
\hline Cobb angle & 18 & $25.4 \pm 3.5$ & 48 & $25.5 \pm 3.5$ & 0.938 \\
\hline \multicolumn{6}{|l|}{ State anxiety } \\
\hline Patient & 19 & $46.0 \pm 11.2$ & 49 & $43.9 \pm 8.9$ & 0.404 \\
\hline Mother & 16 & $48.9 \pm 8.2$ & 38 & $49.8 \pm 12.8$ & 0.790 \\
\hline Father & 4 & $45.3 \pm 7.2$ & 15 & $44.7 \pm 8.3$ & 0.905 \\
\hline \multicolumn{6}{|l|}{ Trait anxiety } \\
\hline Patient & 19 & $42.2 \pm 7.8$ & 49 & $41.7 \pm 8.5$ & 0.826 \\
\hline Mother & 16 & $45.4 \pm 5.8$ & 38 & $45.0 \pm 7.5$ & 0.845 \\
\hline Father & 4 & $42.3 \pm 5.4$ & 15 & $39.4 \pm 7.4$ & 0.462 \\
\hline \multicolumn{6}{|c|}{ Beck Depression Inventory } \\
\hline Patient & 19 & $5.6 \pm 6.6$ & 48 & $4.5 \pm 4.6$ & 0.420 \\
\hline Mother & 16 & $4.1 \pm 4.2$ & 36 & $6.6 \pm 6.5$ & 0.173 \\
\hline Father & 4 & $6.3 \pm 1.0$ & 15 & $3.3 \pm 5.1$ & 0.269 \\
\hline \multicolumn{6}{|l|}{ SRS-22 } \\
\hline Function & 18 & $4.9 \pm 0.1$ & 43 & $4.9 \pm 0.2$ & 0.635 \\
\hline Pain & 18 & $4.7 \pm 0.4$ & 43 & $4.7 \pm 0.4$ & 0.968 \\
\hline Image & 18 & $3.8 \pm 0.6$ & 43 & $3.9 \pm 0.6$ & 0.781 \\
\hline Mental health & 18 & $3.8 \pm 0.6$ & 43 & $4.1 \pm 0.5$ & 0.435 \\
\hline Management & 15 & $3.8 \pm 0.6$ & 34 & $3.5 \pm 0.6$ & 0.130 \\
\hline Sub-total & 18 & $4.4 \pm 0.3$ & 43 & $4.4 \pm 0.3$ & 0.711 \\
\hline Total & 15 & $4.3 \pm 0.3$ & 34 & $4.3 \pm 0.3$ & 0.707 \\
\hline
\end{tabular}


Table II. Characteristics of patients who expressed desired treatment.

(a) Sociodemographics

\begin{tabular}{|c|c|c|c|}
\hline & $\begin{array}{c}\text { Brace } \\
(\mathrm{n}=18)\end{array}$ & $\begin{array}{l}\text { Observation } \\
\qquad(\mathrm{n}=31)\end{array}$ & p-value \\
\hline Age (years) & $12.4 \pm 0.9$ & $12.7 \pm 1.2$ & 0.275 \\
\hline Gender & & & 0.616 \\
\hline Male & $1(5.6)$ & $3(9.7)$ & \\
\hline Female & $17(94.4)$ & $28(90.3)$ & \\
\hline Educational level & & & 0.812 \\
\hline Grade 5-6 & 7 (38.9) & $11(35.5)$ & \\
\hline Grade 7-11 & $11(61.1)$ & $20(64.5)$ & \\
\hline Height (cm) & $153.7 \pm 5.4$ & $155.4 \pm 5.8$ & 0.311 \\
\hline Weight (kg) (2 missing) & $41.0 \pm 8.7$ & $42.5 \pm 8.6$ & 0.567 \\
\hline BMI (kg/m²) (2 missing) & $17.3 \pm 2.9$ & $17.5 \pm 3.3$ & 0.802 \\
\hline Arm span (cm) & $154.6 \pm 7.1$ & $156.1 \pm 7.1$ & 0.478 \\
\hline Monthly household income (HK\$) & & & 0.953 \\
\hline$<2000$ & $1(5.6)$ & $0(0)$ & \\
\hline 2000-5999 & $0(0)$ & $2(6.5)$ & \\
\hline $6000-9999$ & $3(16.7)$ & $2(6.5)$ & \\
\hline $10000-14999$ & $1(5.6)$ & $2(6.5)$ & \\
\hline 15000-19999 & $1(5.6)$ & $10(32.3)$ & \\
\hline 20000-24999 & $3(16.7)$ & $3(9.7)$ & \\
\hline 25000-29999 & $2(11.1)$ & $1(3.2)$ & \\
\hline 30000-39999 & $3(16.7)$ & $2(6.5)$ & \\
\hline 40000-59999 & $2(11.1)$ & $3(9.7)$ & \\
\hline$\geq 60000$ & $2(11.1)$ & $6(19.4)$ & \\
\hline Gestational age (weeks) & $39.7 \pm 1.3$ & $39.3 \pm 1.1$ & 0.267 \\
\hline Maternal age (years) & $28.2 \pm 5.1$ & $31.1 \pm 4.7$ & 0.056 \\
\hline Breastfeeding & & & 0.989 \\
\hline No & $13(72.2)$ & $21(67.7)$ & \\
\hline Yes & $5(27.8)$ & $8(25.8)$ & \\
\hline Do not know / Missing & $0(0)$ & $2(6.5)$ & \\
\hline Smoking among family members & & & 0.159 \\
\hline No & $8(44.4)$ & $19(61.3)$ & \\
\hline Yes & $10(55.6)$ & $10(32.3)$ & \\
\hline Do not know / Missing & $0(0)$ & $2(6.5)$ & \\
\hline Alcohol drinking among family members & & & 0.708 \\
\hline No & $9(50.0)$ & $17(54.8)$ & \\
\hline
\end{tabular}




\begin{tabular}{lccc}
\hline Yes & $8(44.4)$ & $12(38.7)$ & \\
Do not know / Missing & $1(5.6)$ & $2(6.5)$ & \\
Family history of scoliosis & & & 0.982 \\
No & $11(61.1)$ & $18(58.1)$ & \\
Yes & $3(16.7)$ & $5(16.1)$ & \\
Do not know / Missing & $4(22.2)$ & $8(25.8)$ & \\
\hline
\end{tabular}

Data are mean \pm standard deviation for continuous variables or number (\%) for categorical variables

(b) Curvature and quality of life measures

\begin{tabular}{|c|c|c|c|c|c|}
\hline & \multicolumn{2}{|c|}{$\begin{array}{c}\text { Brace } \\
(\mathrm{n}=18)\end{array}$} & \multicolumn{2}{|c|}{$\begin{array}{c}\text { Observation } \\
(\mathrm{n}=31)\end{array}$} & \multirow[b]{2}{*}{ p-value } \\
\hline & $\mathrm{n}$ & mean $\pm \mathrm{SD}$ & $\mathrm{n}$ & mean $\pm \mathrm{SD}$ & \\
\hline Cobb angle & 18 & $26.4 \pm 4.1$ & 30 & $24.9 \pm 3.0$ & 0.160 \\
\hline \multicolumn{6}{|l|}{ State anxiety } \\
\hline Patient & 18 & $44.9 \pm 10.2$ & 31 & $43.3 \pm 8.1$ & 0.532 \\
\hline Mother & 15 & $53.5 \pm 13.1$ & 23 & $47.5 \pm 12.2$ & 0.161 \\
\hline Father & 4 & $37.5 \pm 12.5$ & 11 & $47.4 \pm 4.7$ & 0.141 \\
\hline \multicolumn{6}{|l|}{ Trait anxiety } \\
\hline Patient & 18 & $41.6 \pm 9.1$ & 31 & $41.7 \pm 8.2$ & 0.968 \\
\hline Mother & 15 & $45.33 \pm 8.4$ & 23 & $44.7 \pm 7.0$ & 0.808 \\
\hline Father & 4 & $37.5 \pm 9.3$ & 11 & $40.1 \pm 7.0$ & 0.539 \\
\hline \multicolumn{6}{|c|}{ Beck Depression Inventory } \\
\hline Patient & 17 & $4.8 \pm 4.8$ & 31 & $4.3 \pm 4.5$ & 0.716 \\
\hline Mother & 15 & $9.0 \pm 8.3$ & 21 & $4.9 \pm 4.3$ & 0.079 \\
\hline Father & 4 & $2.5 \pm 3.8$ & 11 & $3.6 \pm 5.6$ & 0.717 \\
\hline \multicolumn{6}{|l|}{ SRS-22 } \\
\hline Function & 15 & $5.0 \pm 0.1$ & 28 & $4.9 \pm 0.2$ & 0.259 \\
\hline Pain & 15 & $4.7 \pm 0.3$ & 28 & $4.7 \pm 0.5$ & 0.712 \\
\hline Image & 15 & $4.1 \pm 0.5$ & 28 & $3.7 \pm 0.6$ & 0.035 \\
\hline Mental health & 15 & $4.2 \pm 0.6$ & 28 & $4.1 \pm 0.5$ & 0.400 \\
\hline Management & 10 & $3.8 \pm 0.7$ & 24 & $3.5 \pm 0.6$ & 0.213 \\
\hline Sub-total & 15 & $4.5 \pm 0.3$ & 28 & $4.4 \pm 0.3$ & 0.112 \\
\hline Total & 10 & $4.4 \pm 0.3$ & 24 & $4.3 \pm 0.3$ & 0.232 \\
\hline
\end{tabular}


Table III Determinants of acceptance to randomization and choosing brace treatment, by stepwise logistic regression.

\begin{tabular}{|c|c|c|c|}
\hline & \multicolumn{3}{|c|}{$95 \%$} \\
\hline & Odds ratio & confidence interval & $\boldsymbol{P}$ \\
\hline \multicolumn{4}{|c|}{ Determinants of acceptance to randomization $(n=68)$} \\
\hline Patient's height (cm) & 0.90 & $(0.81-0.995)$ & 0.040 \\
\hline Monthly household income & 0.75 & $(0.57-0.98)$ & 0.032 \\
\hline \multicolumn{4}{|c|}{ Determinants of choosing brace treatment $(n=49)$} \\
\hline Satisfaction with self-image & 4.1 & $(1.10-15.0)$ & 0.035 \\
\hline
\end{tabular}


Fig. 1

Patient disposition. The treatment in a square bracket is the one on the randomization schedule and that otherwise is the actual.

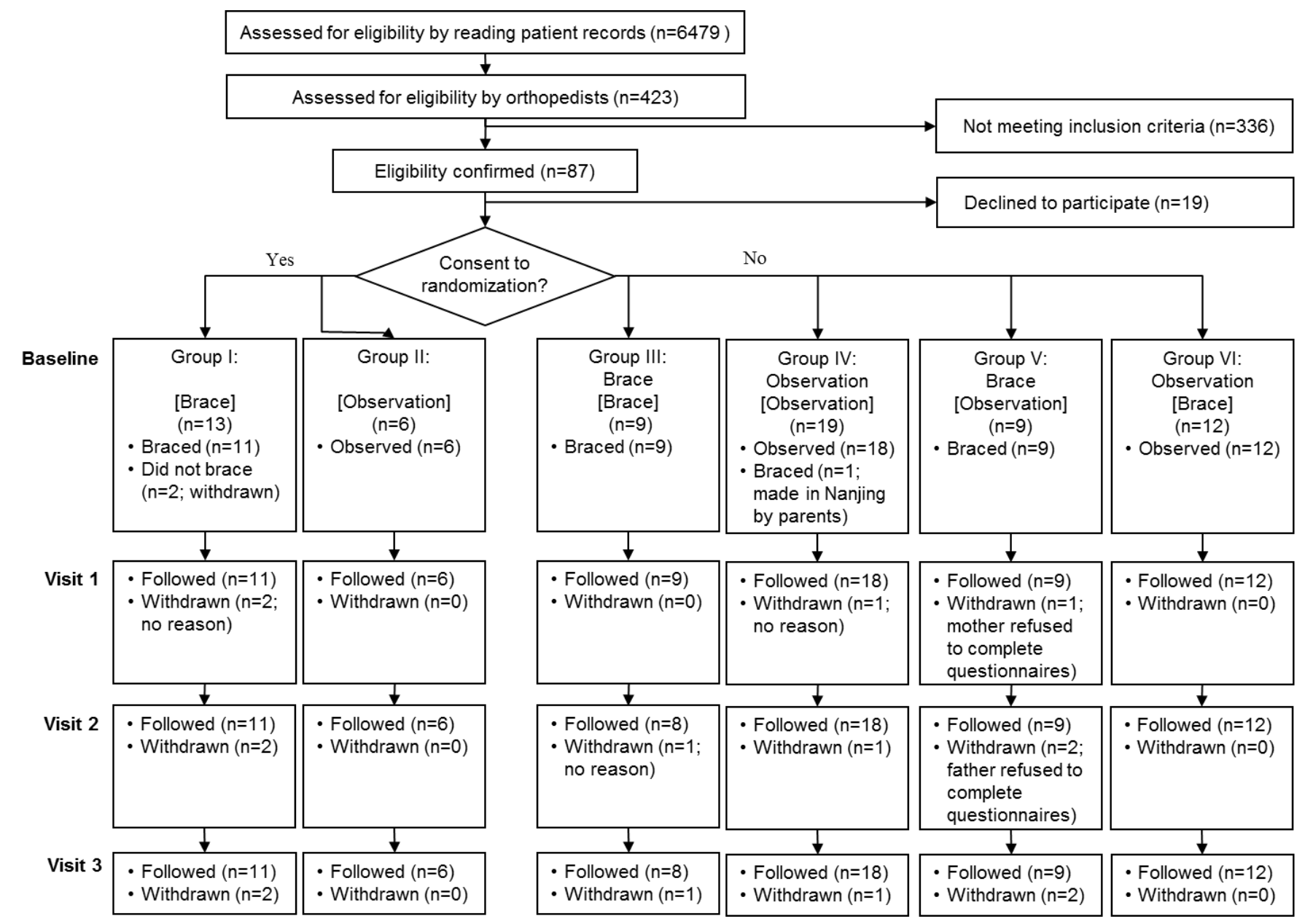

